\title{
Implicaciones del VIH/SIDA en la biografía de varones con prácticas homoeróticas en la Ciudad de México
}

\author{
Gabriel Gallego-Montes, D en Est de Pobl.(1)
}

\begin{abstract}
Gallego-Montes G. Implicaciones del VIH/SIDA en la biografía de varones con prácticas homoeróticas en la Ciudad de México. Salud Publica Mex 2010;52:14|-147.
\end{abstract}

\begin{abstract}
Resumen
Objetivo. Determinar los cambios en la configuración biográfica de un conjunto de varones con prácticas homoeróticas en la era del sida. Material y métodos. Se aplicó una encuesta retrospectiva durante el primer semestre de 2006 a una muestra intencional de 250 varones en la Ciudad de México. Con ellos fue posible reconstruir 633 historias de pareja ocurridas en diferentes momentos del tiempo. Por otro lado, tomando como base el comportamiento epidemiológico del VIH/SIDA en México, se construyeron tres cohortes de emparejamiento: "antes del sida" 1970-1984; 1985-1995 o periodo de "crecimiento exponencial" y 1996-2005 o de "estabilización" de la epidemia. Resultados. Durante el periodo en estudio se encontró una menor exclusividad sexual y una menor duración en las relaciones de pareja, entre cohortes de emparejamientos. Este proceso ha sido documentado en otros lugares y se le ha llamado de "distensión sexual". Conclusiones. El estudio aporta elementos para una historia social de la epidemia y el mejoramiento de políticas públicas de prevención en torno al VIH/SIDA.
\end{abstract}

Palabras clave: VIH/SIDA; homosexualidad masculina; historia; sexualidad; México

\section{Gallego-Montes G.}

Implications of aids on the biography of men

with homoerotics practices in Mexico City.

Salud Publica Mex 2010;52:14I-147.

\begin{abstract}
Objective.To determine the changes in the biography of men with homoerotic practices in the times of AIDS. Material and Methods. A retrospective (biographical) survey was administered during the first semester of 2006 to 250 gay/ homosexual men in Mexico City. Based on this survey, 633 couple stories, taking place in different time periods, were reconstructed. In addition, taking into account the epidemiologic behavior of HIV-AIDS in Mexico, three couple cohorts were defined as:"before AIDS" 1970-1984; period of "exponential growth" 1985-1995; and "stabilization period" 1996-2005.The 633 stories are distributed along these time-frames, taking the starting date of each relationship as the criterion. Results. During the period of this study, less sexual exclusivity and shorter duration of sexual relationships were found among couple cohorts. The latter process has been documented elsewhere and is called of "sexual distension". Conclusions. The research allows for understanding the social history of the epidemic and as well as its implications for HIV-AIDS prevention policies.
\end{abstract}

Key words: HIV;AIDS; homosexuality; men; history; sexuality; Mexico

(I) Universidad de Caldas. Colombia.

Fecha de recibido: 29 de julio de 2009 - Fecha de aceptado: 27 de noviembre de 2009 Solicitud de sobretiros: Dr. Gabriel Gallego Montes. Departamento de Estudios de Familia, Universidad de Caldas. Calle 65 No 26-10, Manizales, Colombia.

Correo electrónico: gabriel.gallego@ucaldas.edu.co 
L as culturas sexuales, ya sean hegemónicas o disidentes, no pueden comprenderse actualmente sin incluirse en el debate la aparición del VIH/SIDA a principios de la década de los ochenta del siglo XX. El sida, como hecho sociocultural, está transformando de manera contradictoria y antagónica ${ }^{1}$ la concepción del cuidado, la intimidad, el emparejamiento y el riesgo en los encuentros íntimos, especialmente relacionado con las prácticas sexuales y el amor entre varones. Para Dowsett, las culturas sexuales están cambiando rápidamente en los países en desarrollo y el VIH/SIDA está actuando como catalizador de estos cambios. ${ }^{2} \mathrm{Sin}$ embargo, en los países latinoamericanos buena parte de la dimensión de estos cambios es desconocida, especialmente desde el punto de vista sociocultural y demográfico, o bien, se carece de las evidencias empíricas suficientes que permitan definir la magnitud y dirección de esos cambios. En México, el mayor conocimiento que se tiene sobre VIH/SIDA es principalmente de carácter epidemiológico.

Tomando como punto de partida la historia de la respuesta institucional frente al VIH/SIDA ${ }^{3}$ y el comportamiento epidemiológico de la enfermedad, ${ }^{4-7}$ es posible entonces proponer tres cortes temporales que permiten identificar a grosso modo los cambios y permanencias en la sexualidad en varones con prácticas homoeróticas de la Ciudad de México.*

El primero de estos cortes se denominará “antes del sida" (1970-1984), período que transcurre desde Stonewall Inn en Nueva York y el nacimiento del Gay Liberation Movement hasta la aparición de los primeros casos de sida a inicios de los años ochenta. En México, el movimiento de liberación homosexual recoge la experiencia de varios movimientos sociales fortalecidos por las revueltas de mayo de 1968. Para finales de la década de los setenta se constituía como un movimiento en expansión con una variada organización interna;,8, en 1979 se organiza la primera marcha del orgullo homosexual en México. ${ }^{9}$ Desde la narrativa y la producción cultural existía en estos años una importante producción literaria homoerótica, ${ }^{9}$ con José Joaquín Blanco y Luis Zapata entre sus máximos exponentes. La publicación del ensayo de Fratti y Batista Liberación Homosexual en 1971 constituyó el primer documento crítico sobre la homosexualidad en la sociedad mexicana. ${ }^{10}$

El segundo momento, llamado de "crecimiento exponencial", abarca el período de tiempo entre los

\footnotetext{
* Al igual que Guillermo Núñez, ${ }^{8}$ se entiende aquí al homoerotismo como el erotismo entre personas del "mismo sexo biológico".
}

años 1985 a 1995, década donde se crea toda una red de respuesta institucional frente al VIH/SIDA a raíz del aumento de la epidemia. ${ }^{3}$ Durante este período se da en el México urbano un afianzamiento del discurso gay que había tenido sus orígenes en el período anterior cuando se crearon las condiciones de posibilidad para la movilización, la asunción pública de sexualidades no heterosexuales y de nuevas formas de relacionarse íntimamente para vivir la experiencia homoerótica, especialmente el emparejamiento y la convivencia en pareja.

El tercer período, que va desde el año 1996-2005 o de "estabilización de la epidemia", coincide con la entrada al mercado de los antirretrovirales (ARV) y la consecuente disminución de las tasas de mortalidad y el aumento en la esperanza y calidad de vida de los portadores del virus. ${ }^{4-7}$ Desde el plano académico emergen las masculinidades como un nuevo campo de estudio, pero carente de una visión articulada entre varones y VIH/SIDA; de igual manera, se comienzan a escuchar las primeras voces acerca de la diversidad sexual. ${ }^{8}$ Para efectos prácticos, este período de tiempo se divide en dos momentos: $1996-2000$ y 2001-2005.

La investigación que da origen a este artículo parte del doble planteamiento construccionista para comprender los procesos sociales. ${ }^{11}$ Es decir, las biografías de los varones con prácticas homoeróticas están afectadas por la dinámica de la epidemia del VIH y a su vez afectan a la misma; los comportamientos sexuales se transforman y dejan huella en la historia personal, de ahí que el objetivo de la investigación consiste en determinar los cambios en las biografías sexuales de un conjunto de varones con prácticas homoeróticas en la era del sida.

Una biografía sexual se entiende como un proceso acumulativo de eventos y vivencias sexuales, que tiene como punto de partida la primera relación sexual, heterosexual u homoerótica, y que se vuelve complejo a partir de las múltiples interacciones sociosexuales que un individuo establece a lo largo de su vida. ${ }^{12-14}$ Estas interacciones están permeadas por la matriz sexo/ género, la posición socioeconómica y las características étnicas en contextos de mayor o menor homofobia, tanto interna como externa. Por relación de pareja se entiende el vínculo erótico-afectivo establecido entre dos varones con una duración igual o superior a tres meses, con o sin corresidencia. ${ }^{12,13,15}$

Las biografías no solamente remiten al tiempo individual, sino que articulan el tiempo social y el tiempo histórico. El VIH/SIDA actúa como una variable básica que permite entender la biografía sexual de un sujeto particular, en un contexto sociohistórico más amplio tal y como se explora en este artículo, es decir, los últimos 35 años de historia en la cultura sexual de la Ciudad de México. 


\section{Material y métodos}

Durante el primer semestre de 2006, una encuesta biográfica o retrospectiva denominada Iniciación sexual, trayectorias de emparejamiento y vida en pareja en varones con prácticas homoeróticas en la Ciudad de México fue aplicada a una muestra intencional de 250 hombres, de entre 16 y 55 años de edad, pertenecientes a una clase media altamente escolarizada donde $72.8 \%$ reportó tener o estar cursando estudios universitarios a nivel licenciatura. ${ }^{9}$

A todos los varones que participaron en la investigación se les informó de manera previa a la entrevista los propósitos y la utilidad de la información, la confidencialidad y el tratamiento estadístico de los datos, todo esto dentro del marco del consentimiento informado.

Para el entramado de la muestra se utilizaron cuatro vías de aproximación a los varones: conocidos del entrevistador $(22 \%)$, bola de nieve saturada en tres contactos por informante (44.8\%), abordaje directo en zonas conocidas de "ligue" sociosexual entre hombres $(26.4 \%)$ y contacto por internet $(6.8 \%)$.

El uso del método biográfico posibilitó reconstruir 633 relaciones de pareja transcurridas en los últimos 35 años en la Ciudad de México. Si bien el diseño del instrumento permitía recuperar cuatro historias por entrevistado, cantidad variable según la edad de los varones, y llegar a una meta de 677 historias, en campo fue posible recuperar $93.5 \%$ del total, debido especialmente a la capacidad de memoria y al olvido de episodios relacionados con el emparejamiento.

Para el manejo estadístico de la información se usó el paquete estadístico SPSS versión 11. El cálculo de las duraciones medianas de las relaciones de pareja se obtuvo a partir de tablas de sobrevivencia en el paquete STATA versión 8.0

\section{Resultados}

Cada uno de los períodos de tiempo identificados, con el VIH/SIDA como referente central, constituye una cohorte de emparejamiento o representa los momentos en que se inician las historias de pareja; la distribución de las diferentes relaciones de pareja por cohorte de emparejamiento y de nacimiento de los entrevistados puede apreciarse en el cuadro I.

El mayor número de historias de pareja (73.6\%) se origina en la década que trascurre entre 1996-2005 o periodo de "estabilización de la epidemia", seguido del número de historias en la época de crecimiento exponencial (20.5\%). Al ver la contribución por grupos de edad, es notable que en cada periodo histórico existe un grupo de edad predominante: $61.5 \%$ de las historias de pareja en el período 1985-1995 es informado por personas mayores de 35 años; en el periodo 1996-2000 la mayor contribución $(54.1 \%$ ) la hace el grupo de entrevistados de entre 25 y 34 años; mientras los más jóvenes hacen el mayor aporte $(47.7 \%)$ en el período 2001-2005.

Es de precisar que existe 3.1\% de historias de pareja iniciadas en el período 1985-1995 de individuos jóvenes nacidos entre 1981-1989. Estas cuatro historias de pareja corresponden a varones nacidos entre 1981-1982 que tuvieron su primera relación de pareja entre los 13 y los 14 años de edad. Por lo tanto, la "precocidad" de estos varones en la construcción de sus trayectorias de emparejamiento genera una ubicación de tales historias al final de la cohorte de emparejamiento 1985-1995. Tal situación es posible dada la "aceleración" que están experimentando los más jóvenes en la construcción de su biografía sexual respecto de los varones mayores de 25 años, la cual se expresa en la edad mediana de entrada al homoerotismo (15.6 años) y en la alta proporción de

Cuadro I

DistRIBUCIÓN PORCENTUAL DE LAS RELACIONES DE PAREJA ENTRE VARONES POR COHORTE DE NACIMIENTO Y EMPAREJAMIMENTO. Ciudad de MÉXICO, 2006

\begin{tabular}{|c|c|c|c|c|c|}
\hline \multirow[b]{2}{*}{ Cohorte de nacimiento } & \multicolumn{5}{|c|}{ Cohorte de emparejamiento } \\
\hline & $\begin{array}{c}\text { Total } \\
(N=633)\end{array}$ & $\begin{array}{c}1970-1984 \\
(N=37)\end{array}$ & $\begin{array}{c}1985-1995 \\
(N=130)\end{array}$ & $\begin{array}{c}1996-2000 \\
(N=183)\end{array}$ & $\begin{array}{c}200 I-2005 \\
(N=283)\end{array}$ \\
\hline $1950-1970$ & 30.0 & 100 & 61.5 & 21.3 & 12.0 \\
\hline $197 \mid-1980$ & 40.9 & 0.0 & 35.4 & 54.1 & 40.3 \\
\hline 1981-1989 & 29.1 & 0.0 & 3.1 & 24.6 & 47.7 \\
\hline Total & 100 & 100 & 100 & 100 & 100 \\
\hline
\end{tabular}


varones que a la edad de 20 años ya había tenido su primera experiencia sexual con otro varón (95.6\%).

La mayor parte de las historias de pareja de las dos cohortes de entrevistados más jóvenes, 16-24 y 25-34 años, se iniciaron en el periodo 2001-2005, con 73.4 y $44 \%$ respectivamente, mientras que en el grupo de 35 y más años el grueso de historias de pareja (42.1\%) se inicia en el período 1985-1995, y muy pocas son las que ocurren en el período 2001-2005 (17.9\%). Esta distribución por grupos de edad se debe a la duración mediana que en cada cohorte de entrevistados han tenido las relaciones de pareja con otros varones, donde es mayor para los entrevistados de 35 y más años, con 23 meses de duración, y menor para aquellos entre los 16-24 años de edad, con 7 meses de duración. Estas experiencias más cortas y fugaces, altamente inestables, pero en monogamia en serie en la construcción de relaciones de pareja con otros varones, conllevan a la mayor acumulación de estas breves historias en ciertas cohortes de emparejamiento, en especial en la última década.

Adicionalmente, la concentración de buena parte de la muestra entre los 16-40 años de edad (87\%) y la estimación de la edad mediana en la que se dio el primer enamoramiento con otro varón (22.3 años) explican también la distribución y concentración en ciertas cohortes de mayores historias de pareja.

\section{Del "ligue" callejero al bar y la internet. Elementos de institucionalización de la vida gay en la Ciudad de México}

La institucionalización de la vida erótica y afectiva entre varones en la era del sida ha implicado la delimitación de lugares concretos y específicos de interacción entre parejas homosexuales, ${ }^{9}$ con una oferta variada de espacios como bares y cafeterías, lugares de ligue, saunas y cuartos obscuros, donde es posible que se produzcan una mayor cantidad de encuentros sexuales. En la Ciudad de México el predominio del bar gay y la internet con fines sociosexuales constituyen una de las expresiones del proceso de institucionalización. El cuadro II permite señalar cómo en los últimos 35 años se ha dado un desplazamiento-consolidación de ciertos lugares como parte de la configuración de una geografía del deseo homoerótico $(p<0,05)$.

Para ilustrar lo anterior, se considerarán dos conjuntos de lugares: el primero está constituido por la calle-el metro-la fiesta, sitios que representan los lugares clásicos del "ligue" entre varones en el período anterior a la aparición del sida, y el segundo, formado por los bares y la internet, refleja la institucionalización del ligue en la era del sida con el establecimiento de fronteras precisas, casi de guetto. El proceso de tránsito de un grupo de lugares a otro refleja adicionalmente la formación de la subcultura gay.

Muy pocos investigadores se han dedicado a investigar el erotismo y la afectividad entre hombres antes de la aparición del sida. En las obras literarias de Luis Zapata y José Joaquín Blanco la calle es el lugar donde trascurren buena parte de las historias de los personajes como el célebre Adonis García, un chichifo (prostituto) de profesión, en El vampiro de la colonia Roma. ${ }^{16}$ Se tiene poca información publicada de la función sociosexual que el sistema de transporte metro ha cumplido para facilitar el "ligue" entre personas del mismo sexo; sólo existe información en ciertos grupos y redes de varones que conocen vagones, horarios y líneas en donde es posible el "ligue" con distintos fines, actividad a la que se le llama coloquialmente "metrear".

Cuadro II

Distribución PORCENTUAL DE LOS LUGARES DE LIGUE ENTRE VARONES POR COHORTE DE EMPAREJAMIMENTO. Ciudad de MÉxico, 2006

\begin{tabular}{|c|c|c|c|c|c|}
\hline \multirow[b]{2}{*}{ Lugares de ligue } & \multicolumn{5}{|c|}{ Cohorte de emparejamiento } \\
\hline & $\begin{array}{c}\text { Total } \\
(N=633)\end{array}$ & $\begin{array}{c}1970-1984 \\
(N=37)\end{array}$ & $\begin{array}{c}1985-1995 \\
(N=130)\end{array}$ & $\begin{array}{c}1996-2000 \\
(N=183)\end{array}$ & $\begin{array}{c}200 I-2005 \\
(N=283)\end{array}$ \\
\hline Bar/café gay & 22.9 & 5.4 & 22.3 & 23.5 & 25.1 \\
\hline Calle & 13.7 & 27.0 & I3.1 & 12.6 & 13.1 \\
\hline Escuela/prepa/universidad & 13.1 & 18.9 & 16.2 & 12.6 & 11.3 \\
\hline Fiesta/reunión & 12.5 & 13.5 & 13.1 & 15.8 & 9.9 \\
\hline Por internet & 8.5 & & & 7.1 & 14.5 \\
\hline Metro & 4.6 & 8.1 & 8.5 & 5.5 & 1.8 \\
\hline Otro & 24.6 & 27.0 & 26.0 & 23.0 & 24.4 \\
\hline Total & 100 & 100 & 100 & 100 & 100 \\
\hline
\end{tabular}


Antes de la irrupción del sida, las formas de "ligue" entre hombres no tenían fronteras espaciales precisas; la literatura científica disponible sobre homosexualidad y la evidencia empírica de estas 37 historias ocurridas en este período de tiempo parecen confirmar tal sentencia. El 95\% de las historia de pareja ocurridas entre 1970-1985 tuvieron como punto de encuentro un lugar no específicamente "para hombres". Si bien desde mediados del siglo XIX han existido baños para señores en la Ciudad de México, ${ }^{17}$ no constituían un escenario propicio para el enamoramiento entre varones, dadas las específicas funciones sociosexuales para las que estaban diseñados, en un ambiente de completa masculinidad; la misma situación sucedía con los bares.

Con el advenimiento del discurso gay se inicia un proceso de institucionalización de la vida eróticaafectiva entre varones con la tendencia de verse reducida esta experimentación a lugares y zonas concretas. Un reflejo de ello es la disminución en la era del sida del "ligue" en la calle, en el metro o en la fiesta y de otras formas no institucionalizadas "de levante" entre hombres. Mientras en la época del ambiente estos lugares representaban la forma como se habían iniciado $48.6 \%$ de las historias de pareja, en el período 2001-2005 su importancia se redujo a 24.8\% $(p<0,05)$.

El proceso de institucionalización del "ligue" tiene al bar gay como su mayor exponente. Para la década de 1985-1995 los bares habían propiciado el inicio de 22.3\% del total de historias de pareja en este periodo; para el lustro 2001-2005, una de cuatro historias de pareja se habían iniciado en algún bar gay de la Ciudad de México. La internet (correo electrónico y chat room gays) constituye, en el último período de tiempo, el medio en el que $14.5 \%$ de las parejas se conocieron e iniciaron una relación.

En general, la institucionalización del "ligue" implica una resignificación y uso de espacios como los "lugares" para el conocimiento de otros varones con los cuales se podría entablar una relación de pareja. Entre el bar y la internet se concentra 40\% del "ligue" entre varones en el último período de tiempo, toda una revolución en materia de desarrollo institucional con fines socio-sexuales.

\section{Exclusividad sexual y duración mediana de las relaciones de pareja entre varones}

El período en estudio muestra cambios significativos en materia de exclusividad sexual y duración mediana en las relaciones de pareja entre varones. Como se aprecia en la figura 1, el período entre 1985-1995 o de "crecimiento exponencial" de la epidemia del sida estuvo marcado por una mayor exclusividad sexual bajo el modelo de pa-

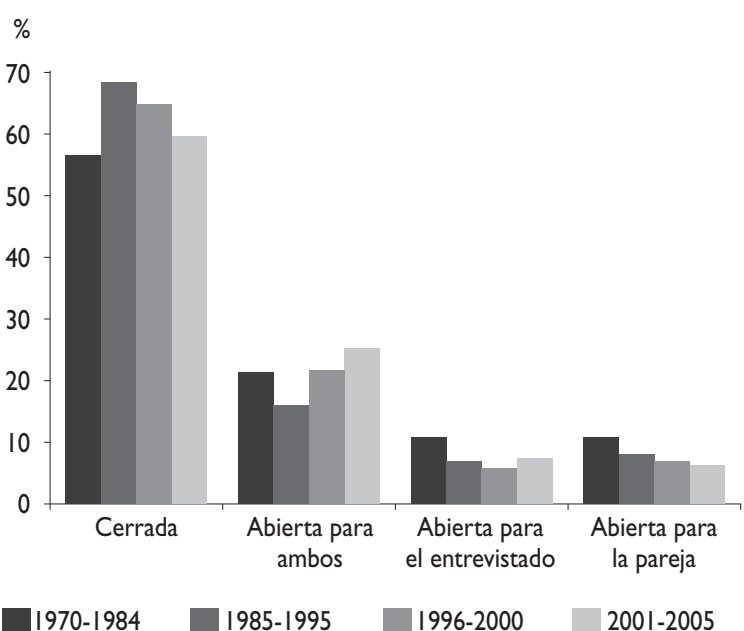

Figura I. Exclusividad sexual en relaciones de pareja ENTRE VARONES POR COHORTES DE EMPAREJAMIENTO. CIUDAD DE MÉXICO, 2006

reja cerrada (68\%) y una disminución en las preferencias por encuentros sexuales con terceras personas, ya sean en relaciones diádicas, tríos o sexo en grupo $(p<0,05)$. La duración mediana de los emparejamientos para este periodo de tiempo (cuadro III) tiende a confirmar tal hallazgo: una relación de pareja sin corresidencia o de "noviazgo" tuvo una duración mediana de 17 meses, y con corresidencia de 57 meses (3.35 veces más tiempo). En comparación con el período anterior, denominado "antes del sida", la emergencia de la epidemia estimuló en este grupo de entrevistados una apuesta por relaciones de pareja más largas y, en la medida de lo posible, con corresidencia; esta estrategia, aunada al modelo de pareja cerrada, constituyó tal vez un blindaje frente al crecimiento exponencial de la epidemia que se dio en esta década.

Las relaciones de pareja que se iniciaron durante la década 1996-2005 apostaron un poco menos por el patrón de pareja cerrada, en relación con los emparejamientos de la década anterior $(p<0,05)$, constituyendo en más de un tercio relaciones abiertas en sus diferentes modalidades: para ambos, para el entrevistado o para su pareja. En el último lustro que abarca este estudio, las parejas abiertas o no exclusivas sexualmente conformaban $40 \%$ del total de acuerdos de pareja que se habían iniciado en este período de tiempo $(p<0,05)$. La duración mediana de las relaciones de pareja también disminuyó; una relación tipo "noviazgo" tuvo una duración mediana de nueve meses en el lustro 19962000 y de seis meses para aquellas relaciones iniciadas entre 2001-2005. Esta menor duración de las relaciones 
Cuadro III

DuRACIÓn MEdiana (EN MESES) DEL CORTEJO, DE LA RELACIÓN DE PAREJA CON Y SIN CORRESIDENCIA Y DE LA CORRESIDENCIA EN CADA COHORTE DE EMPAREJAMIENTO. Ciudad de México, 2006

Cohorte de emparejamiento

Mediana N

1970-1984

\begin{tabular}{lcc} 
Duración cortejo & 0.0 & 37 \\
\hline Duración de la relación sin corresidencia & 16.5 & 26 \\
\hline Duración de la relación con corresidencia & 44.0 & II \\
\hline Duración de la corresidencia & 40.0 & II
\end{tabular}

1985-1995

\begin{tabular}{lcc} 
Duración cortejo & 0.0 & 130 \\
\hline Duración de la relación sin corresidencia & 17.0 & 75 \\
\hline Duración de la relación con corresidencia & 57.0 & 55 \\
\hline Duración de la corresidencia & 47.0 & 55
\end{tabular}

1996-2000

\begin{tabular}{lcc} 
Duración cortejo & 1.0 & 183 \\
\hline Duración de la relación sin corresidencia & 9.0 & 120 \\
\hline Duración de la relación con corresidencia & 35.0 & 63 \\
\hline Duración de la corresidencia & 17.0 & 63
\end{tabular}

$2001-2005$

\begin{tabular}{lcc} 
Duración cortejo & 0.0 & 283 \\
\hline Duración de la relación sin corresidencia & 6.0 & 205 \\
\hline Duración de la relación con corresidencia & 17.5 & 78 \\
\hline Duración de la corresidencia & 10.0 & 78
\end{tabular}

Total

\begin{tabular}{lcc} 
Duración relación de pareja sin corresidencia & 8.0 & 426 \\
\hline Duración relación de pareja con corresidencia & 27.0 & 207 \\
\hline Duración de la corresidencia & 17.0 & 207 \\
\hline Total historias de pareja & 12.0 & 633
\end{tabular}

de pareja en varones altamente monogámicos $(84 \%)$ genera un efecto de monogamia serial y, por lo tanto, de acumulación de historias en el último período de tiempo.

Sin embargo, el comportamiento de la corresidencia tiene un efecto contrario al de la monogamia serial, es decir, existe una mayor acumulación de historias en el último período de tiempo y menor duración de las mismas. El $42 \%$ de las relaciones de pareja que se iniciaron entre 1985-1995 fueron corresidentes y tuvieron una duración mediana de 57 meses; durante el período 19962000 el número de parejas corresidentes se redujo en 8 puntos porcentuales con respecto del período anterior, $\mathrm{y}$ la duración igualmente se redujo a 35 meses. Durante el último período de tiempo, sólo una de cuatro parejas fue corresidente con una duración mediana de 17.5 meses, la mitad de lo que duraban las relaciones que se habían iniciado sólo un lustro antes. * Todo parece indicar que en la era del sida tiende a predominar el noviazgo como forma vinculante.

\section{Discusión}

Las biografías constituyen uno de los elementos básicos en la demografía y los estudios de población, ya que permiten una comprensión compleja y dinámica de ciertos eventos acaecidos en el curso de la vida de un individuo. El uso del enfoque biográfico también permite leer procesos y transformaciones sociales en la vida de las personas, como los ocurridos a raíz de la epidemia del VIH/SIDA.

La periodización propuesta en esta investigación y la ubicación de las historias de pareja entre cohortes permiten identificar el proceso de institucionalización de la vida erótica y afectiva entre varones marcado por dos elementos básicos: un aumento del "ligue" institucional expresado en la preponderancia del bar gay y la internet y una incipiente, pero simbólica y jurídicamente significativa, regulación de la vida en pareja bajo el marco de las sociedades de convivencia. ${ }^{9}$

Estas nuevas condiciones sociales, la llegada de los ARV y el consiguiente aumento en las expectativas y esperanza de vida de los portadores del virus, cambiaron el comportamiento sociocultural frente a la epidemia, en un proceso que ha sido denominado de "distensión sexual". Tal proceso tiene, entre otras manifestaciones, una pérdida relativa del modelo de pareja cerrada y una menor apuesta por relaciones de pareja, reflejada en las duraciones de los emparejamientos. Este proceso de "distensión" también ha sido documentado en otros lugares como San Francisco, ${ }^{18}$ Sao Paulo ${ }^{19}$ y Australia. ${ }^{20}$

La estrategia de monogamia = exclusividad sexual que fue parte de todas las campañas informativas y educativas en torno al VIH/SIDA, en pleno contexto de pánico moral y sexual, ${ }^{21}$ parece haber tenido efectividad pues así lo demuestran las biografías de los varones mayores de 35 años que vivieron el cambio en los patrones de emparejamiento con el surgimiento del VIH/SIDA.

\footnotetext{
* Si bien las relaciones de pareja tienden a durar menos en el curso de vida de las tres cohortes de entrevistados, los datos de la última década de análisis se encuentran afectados por la estructura de edad de la muestra y, por lo tanto, de una mayor acumulación de historias de los jóvenes donde es menos frecuente la corresidencia.
} 
Las políticas públicas en torno al VIH/SIDA tendrían que reconocer la forma como opera el proceso de institucionalización de la vida erótica y afectiva entre varones y diseñar acciones para hacer frente al proceso de distensión sexual.

\section{Referencias}

I. Adam B. Care, intimacy and same-sex partnership in the 21 st century. Curr Sociol 2004; (52)2:265-279.

2. Dowsett G.Yo te enseño el mío si tú me enseñas el tuyo: hombres gay, investigación sobre masculinidad, estudios sobre hombres y sexualidad. En:Amuchástegui A, Szasz I, Coords. Sucede que me canso de ser hombre. Relatos y reflexiones sobre hombres y masculinidad en México. México: El Colegio de México, 2005.

3. Magis C, Parrini R. Nuestra Epidemia, el sida en México 1983-2002. En: SIDA:Aspectos de salud pública. México: INSP-CENSIDA, 2006.

4. Ponce de León S. El principio de la epidemia en México. Rev Invest Clin 2004; (56) 2: I24-I 25.

5. Magis C, Bravo-García E, Uribe P. Dos décadas de la epidemia del SIDA en México [monografía en internet]. México: CONASIDA, 2003.

[Consultado 2007, mayo]. Disponible en: http:/www.salud.gob.mx / conasida/ pdf/ dosdecadas.pdf

6. Tapia R, Bravo-García E, Uribe P. Evolución de la epidemia del SIDA en México. En:Alarcón D, Ponce de León S. Compil. El sida en México, veinte años de la epidemia. México: El Colegio Nacional, 2003.

7. Saavedra J, Bravo-García E. Panorama delVIH-SIDA en el 2006. En: SIDA: Aspectos de salud pública. México: INSP-CENSIDA, 2006.

8. Núñez G. Reconociendo los placeres, deconstruyendo las identidades. Antropología, patriarcado y homoerotismo en México. Desacatos Revista de Antropología Social 200 I; 6: 15-34.
9. Gallego G. Patrones de iniciación sexual y trayectorias de emparejamiento entre varones, una mirada biográfica-interaccional en el estudio de la sexualidad (Tesis). México: El Colegio de México, 2007.

10. Fratti G, Batista A. Liberación homosexual. México: Editorial Posada, 1984.

I I. Giddens A. The constitution of society: outline of the theory of structuration. Cambridge: Polity, 1984.

12. Heilborn ML, Cabral C. As trajetórias homo-bissexuais. En: Heilborn $\mathrm{ML}$, et al, (orgs.) $\mathrm{O}$ aprendizado da sexualidade, reprodução e trajetórias sociais de jovens brasileiros. Rio de Janeiro: Universitária, 2006.

13. Knauth D, Gomes C, Fachel A, Fachel J.As trajetórias afectivo-sexuais: encontros, uniões e separação. En: Heilborn ML, et al, (coords.) O aprendizado da sexualidade, reprodução e trajetórias sociais de jovens brasileiros. Rio de Janeiro: Universitária, 2006.

14. Plummer K. Telling sexual stories. Londres: Routledge, 1995. 15. Juárez F, Castro T. Partnership and Sexual Histories of Adolescent Males in Brazil: Myths and Realities. Ponencia presentada en la reunión anual de la Asociación Americana de Población, 2004; Boston, USA. 16. Zapata L. El vampiro de la colonia roma. Las aventuras, desventuras y sueños de Adonis García. México: Grijalbo, 1979.

17. Macías-González V. Entre lilos limpios y sucias sarasas: la homosexualidad en los baños de la ciudad de México, 1880-1910. En: Collado MC. Coord. Miradas recurrentes II, la Ciudad de México en los siglos XIX y XX. México: Instituto Mora-UAM, 2004.

18. Shenon N, Crosby M.Ambivalent tales of HIV disclosure in San Francisco. Soc Sci Med 2004; (58) I I:2 I 05-2 I I8.

19. Parker R. Beneath the equator: Cultures of desire, male homosexuality and emerging gay communities in Brazil. Nueva York: Routledge, 1999. 20. Dowsett G. Some Considerations on Sexuality and Gender in the Context of AIDS. Reprod Health Matters 2003; (II)22: 2I-29.

21.Aggleton P, Parker R. Estigma y discriminación relacionados con el VIHSIDA: un marco conceptual e implicaciones para la acción, documento de trabajo \# 9, Programa de salud reproductiva y sociedad. México: El Colegio de México, 2002. 